\title{
Global entrainment in the brain-body-environment: retrospective and prospective views
}

\author{
Gentaro Taga ${ }^{1}[$ \\ Received: 15 July 2021 / Accepted: 25 September 2021 / Published online: 11 October 2021 \\ (c) The Author(s), under exclusive licence to Springer-Verlag GmbH Germany, part of Springer Nature 2021
}

\begin{abstract}
We celebrate the 60th anniversary of Biological Cybernetics. It has also been 30 years since "Self-organized control of bipedal locomotion by neural oscillators in unpredictable environment" was published in Biological Cybernetics (Taga et al. in Biol Cybern 65(3):147-159, 1991). I would like to look back on the creation of this paper and discuss its subsequent development and future perspectives.
\end{abstract}

Keywords Global entrainment $\cdot$ Rhythm $\cdot$ Neural oscillator $\cdot$ CPG $\cdot$ Bipedal locomotion $\cdot$ Motor development $\cdot$ Brain development

\section{Entrainment and motor control}

Norbert Wiener described the entrainment of nonlinear oscillators and the control of human motion in Cybernetics (1961). However, he may not have been aware of the relationship between the two. In 1988, I joined the laboratory of Professor Hiroshi Shimizu of the University of Tokyo as an undergraduate student. Prof. Shimizu was interested in the synchronization phenomenon of firefly populations and EEG described in Cybernetics (1961) and conducted theoretical research on the entrainment of nonlinear oscillators with Yamaguchi et al. (1981). Furthermore, he proposed a pioneering hypothesis that entrainment plays an important role in the creation and coding of information in the brain and constructed a model for pattern recognition (Shimizu et al. 1985). He argued that an important function of the brain is not to store information obtained from the environment by learning, but to create new information through dynamic cooperation between the different brain components. At that time, molecular biology was rapidly developing, and many studies focused on identifying specific molecules related to specific functions. In neurophysiology, the focus was on identifying neuronal activity associated with a particular function

Communicated by Benjamin Lindner.

Gentaro Taga

taga@p.u-tokyo.ac.jp

1 Graduate School of Education, The University of Tokyo, 7-3-1 Hongo, Bunkyo-ku, Tokyo 113-0033, Japan or stimulation. However, even if the elements of biological systems are identified, it is not clear what principle makes it possible to maintain a living state and create new information as a whole system, particularly one in which a large number of heterogeneous elements interact in a complex manner.

I was fascinated by Prof. Shimizu's idea that it is necessary to pursue the problem of information generation in the brain that is based on self-organization in a nonlinear nonequilibrium system. Therefore, I started to study coupled neural oscillators with Hiroshi Hasegawa, who was also a new student in Shimizu's laboratory, under the enthusiastic guidance of Yoko Yamaguchi. Around that time, a second neural network boom began to develop models for the brain that were composed of formal neuron units (Amari 1977; Kohonen 1982; Hopfield and Tank 1985), and only a few studies had focused on the temporal aspects of neural dynamics in relation to functioning of the brain (Shimizu et al. 1985; von der Malsburg and Schneider 1986). Walter Freeman visited Shimizu's laboratory and gave a lecture on the chaos of neural dynamics (Freeman 1987), by which I was extremely intrigued. One day, Yoko Yamaguchi excitedly told me that evidence to support the theory of pattern recognition by entrainment of nonlinear oscillators (Shimizu and Yamaguchi 1987) had finally been found. In this experiment, it was demonstrated that entrainment of cortical oscillations is involved in the coherent perception of visual objects (Gray and Singer 1989). While the idea that the synchronization phenomenon of gamma oscillations plays an important role in brain function-acquired citizenship in neuroscience, I was 
stuck on one serious question. Most models of the brain focused only on receiving and recognizing information from the environment and did not have a body that interacted with the environment.

I noticed a phenomenon in walking living beings. For example, in both the six-legged walking of insects and quadrupedal walking of mammals, coordinated movement is realized by periodically moving each leg and maintaining a constant phase relationship between them. Humans also achieve stable walking by coordinating many muscles in the left and right legs. As their neural mechanism, Sten Grillner revealed that the spinal cord has a central pattern generator (CPG) that produces basic gait patterns in cats and lampreys (Grillner 1985). Therefore, the generation of gait with a CPG can be understood as pattern generation in a coupled nonlinear oscillator system, which seems to be more straightforward than oscillatory processing in visual perception. Scott Kelso developed an approach on the basis of Haken's synergetics that captures the generation and change of motor patterns as a self-organizing phenomenon (Haken et al. 1985). ${ }^{1}$ In 1989, Prof. Shimizu had the opportunity to host an international symposium at the University of Tokyo. I asked him to add Grillner and Kelso to the list of invited speakers, and they agreed to come to Tokyo. I was deeply influenced by their lectures and conversations with them in person. However, the relationship between the brain and body remained unclear. While the isolated CPG spontaneously produced activities corresponding to motor patterns, the actual activity of the CPG was never observed when the animals were walking. Furthermore, actual locomotion requires continuous adaptation to changing environments. Thus, it was not clear how a CPG controls locomotion in real-world settings. Therefore, I constructed a model for the neural system, the body, and the environment as a dynamical system. It was a great challenge to configure the motor control system as a self-organizing system.

Wiener described the concept of feedback control in cybernetics, which was born out of consideration for human motor control. Subsequently, the control theory evolved independently of the elucidation of the human motion control mechanism and led to machine control and robotics. However, control of bipedal locomotion of human-like robot is one of the most difficult problems within the framework of modern control theories. Since the interaction between the ground and the legs changes from moment to moment, sticking to realizing the motion trajectory as planned in advance was not a good strategy. In contrast to this, the body has intrinsic dynamics: The motion of the body's center of gravity with respect to the center of pressure exerted on the feet can be regarded as that of inverted pendulum, and the motion

\footnotetext{
1 The Haken-Kelso-Bunz model has been most recently reviewed in another retrospective piece in this journal (Kelso 2021).
}

of a swinging limb can be regarded as that of a coupled pendulum. If a gentle inclination of the ground is provided, passive walking is possible without any control. Therefore, I came up with the working hypothesis that if we consider the differential equations for a neural system consisting of neural oscillators and the equations of motion of the body as a dynamical system, as well as provide the appropriate interactions and parameters, stable walking will emerge as an attractor state. In other words, I removed the premise that the neural system is equal to the control system, and that the body is equal to the controlled system. This is the concept of global entrainment in the brain-body-environment.

\section{Fall down seven times and get up eight times}

I attempted to simulate a model for bipedal locomotion on a personal computer. It took hours to calculate only a few steps of the motion, and it was difficult to test many different models and parameters. By using a supercomputer operated by the time-sharing system of the University of Tokyo, it became possible to try many models with many parameters. Initially, I tried to create a model of six legs or four legs, but I thought that it would be better to solve the instability problem in a system where the difference between failure and success is clear, as in bipedal walking. However, no matter how many times I tried, falling down continued for about half a year. It was like the Japanese proverb, "fall down seven times and get up eight times."

One day, I got out of the laboratory and visited my hometown. When I looked at the blue shining sea by the tower of an old castle, a thought suddenly came to my mind. The bipedal walking pattern of the crane is quite different from that of humans, depending on the structure of the body. In my simulation, there were not enough constraints to prevent the knee joint from bending in the opposite direction. I immediately returned to the laboratory and added a damper to the knee of the model. Then, the model suddenly started to walk forever as long as the motion converged to a stable limit cycle. It was April 1990. I thought I finally achieved integration of Wiener's two issues, namely entrainment and control, and coined the term global entrainment.

After that, I encountered several unexpected features of the model, one after another. The model was robust to mechanical perturbations in the body. The model also changed the step-length of walking, depending on the slope of the ground. What was even more surprising was that the gait pattern changed from walking to running when the input parameter to the CPG was increased. It is often criticized that a person can create a model in any way to reproduce the results of an experiment or a specific phenomenon, which never leads to true proof. However, what is important here is that locomo- 
tion cannot be prescribed in advance, and I can test what kind of movement the system generates in a situation that is open to an unpredictable environment.

Biological Cybernetics was a dream stage for me to show my work. Thus, I compiled these results with Yoko Yamaguchi and Prof. Shimizu and decided to submit the manuscript to Biological Cybernetics. However, submitting to Biological Cybernetics was inauspicious for Shimizu's laboratory, because a previously submitted paper on the model for visual pattern recognition based on the entrainment of nonlinear oscillators had been rejected. We sent the paper to Masao Ito, who was a world authority on neurophysiology of the cerebellum and motor control and an editorial board member of Biological Cybernetics. After a while, along with the peer-reviewed comments written in Japanese, we received a letter of rejection. This is because the control principle of the proposed model was not clear from the viewpoint of robotic research on bipedal locomotion. Despite this, we wrote a counter-argument in Japanese and sent a modified version. A few months later, we received a letter of acceptance from the editor-in-chief.

After the publication of this paper (Taga et al. 1991), we received many encouraging comments such as "provoking" from Sten Grillner, "seminal work" from Walter Freeman, and "just beautiful" from Esther Thelen. In domestic meetings, Ichiro Tsuda provided thoughtful comments from the perspective of neurodynamics (Tsuda 1992). Kunihiko Kaneko suggested the possibilities of further developing the model within the framework of complex systems (Kaneko 1990). Mitsuo Kawato provided constructive criticism regarding how the brain-body system may take into account the internal model for motor control (Kawato et al. 1987). At that time, it was customary to request reprints from authors as postcards after publication, and postcards sent from all over the world on a daily basis showed the magnitude of the response to the study. In 1993, I spent a short time in Sten Grillner's laboratory and discussed it with Örjan Ekeberg, Tom Wadden, and Hans Forssberg. I also visited Valentino Braitenberg, Gregor Schöner, Holk Cruse, and Knoek van Soest in Europe and Reggie Edgerton, Alan Garfinkel, Kenji Doya, Terrence Sejnowski, Jim Collins, and Trevor Drew in North America. Valuable discussions with all of these people helped me to think about the next steps in my research.

\section{Revealing mechanisms for human locomotion}

After the publication of the first paper, I attempted to develop my research in several directions. The model of the human musculoskeletal system was extended to have three segments for each leg and two segments for the upper body. The neural system was also extended in accordance with the complexity of the body. This made it possible to compare the output of the model with human data of movements and ground reaction forces obtained in biomechanical studies (Winter 1991). I learned about human gait analysis through discussions with Nobutoshi Yamazaki and Shinji Miyazaki. A neural system for locomotor control was designed, and parameter values were chosen to reproduce the basic aspects of human gait. The conductance-based neuron model, the Hodgkin-Huxley equation, consists of hypothetically derived mathematical terms for voltage-gated currents and gate dynamics with parameters fitted to the experimentally obtained data of neurons. Although the entire system for human locomotion is extremely complex compared with the neuronal model, I aimed to build a minimal model that reproduces the essential features of human gait. For about 2 years, I continued constructing and discarding models to obtain finally a satisfactory model that generates stable locomotion. Once the model was proven to be stable, experiments were performed to determine the environmental conditions that destabilize walking and the changes in the neural and/or musculoskeletal system that affect walking. These results were written in two papers and submitted to Biological Cybernetics. However, I received a letter of rejection again with peer-reviewed comments written in Japanese. At that time, Masatoshi Murase, an adviser during my postdoctoral fellowship at Kyoto University, encouraged me to retry the submission. I revised some parts of the manuscript and submitted them with a rebuttal letter. They were then accepted (Taga 1995a,b).

While building a framework to view human locomotion as a self-organizing phenomenon, it is not clear how a cortical mechanism is involved in the anticipatory control of locomotion in a complex environment. Inspired by Trevor Drew's study on cerebral cortex activity in walking cats (Drew 1988) and Patla's study on obstacle avoidance during human walking (Patla et al. 1991), I created a model of walking over obstacles based on visual information (Taga 1998). The essential feature of the model is that the cortex generates discrete movement signals for gait modulation based on both visual information and phase information of efference signals from the CPG. After that, Aymar de Rugy asked me if the model could be used to explain the locomotor pointing task, which requires accurate positioning of the foot on a visible target on the floor during walking. De Rugy stayed at my laboratory for a few months and succeeded in simulating the locomotor pointing properties by coupling visual information and motor pattern generation based on a CPG (de Rugy et al. 2002). This study showed that the affordance theory of ecological psychology and the self-organization theory of motor pattern generation can be integrated. Later, in relation to this issue, psychophysics studies were performed to show a novel optical illusion while walking on a treadmill (Yabe and Taga 2008). 


\section{Robotics}

The elucidation of neural mechanisms for locomotion has had some impact on robotics. How the redundant degrees of freedom are coordinated and how the stability of movements is maintained under a changing environment are common problems for both humans and robots. However, multiple strategies to achieve this may exist. When I was studying at Boston University in 1994, I frequently attended the seminar of Marc Raibert's laboratory at MIT. Raibert had been attracting attention in robotics because he created acrobatic hopping robots using a minimum control strategy to make use of intrinsic body dynamics, such as elastic forces (Raibert 1986). This spirit seems to be alive in the incredible performance of the robots currently being developed by Boston Dynamics. In 1996, the automobile company Honda suddenly released a bipedal walking robot that attracted a great deal of attention. This study showed that it is technically possible to develop a bipedal walking robot that can withstand practical use. Since then, research on bipedal walking robots has increased, and control by a CPG has been an issue to be examined as a control strategy.

I pursued the possibility of applying CPG-based control to humanoid robots with Yasuo Kuniyoshi et al. (Miyakoshi et al. 2000). This effort might have affected the development of the concept of embodiment in cognitive science (Pfeifer and Scheier 2001) and the creation of a new research field, such as cognitive developmental robotics (Asada et al. 2009).

Many control models based on CPGs have been constructed for animals other than humans, such as four-legged animals (Fukuoka et al. 2003), six-legged animals (Kimura et al. 1993), and fish (Ekeberg 1993). These studies not only contribute to the understanding of the neural control of behaviors in animals, but also have an impact on robotics. Active research is being conducted in an area called biorobotics (Ijspeert 2014).

\section{Was the hypothesis of the global entrainment proven in human locomotion?}

To what extent has the global entrainment hypothesis of human locomotion been proven at this time? Since the rehabilitation of walking impaired by spinal cord injury is a medically important issue, the neural mechanism of human walking has been intensively studied. In 1998, it was reported that epidural electrical stimulation of the lumbar spinal cord induces gait-like movement patterns in patients with paralyzed lower limb movements due to spinal cord injury (Dimitrijevic et al. 1998). This study provides the first evidence for the existence of a CPG for walking in the human spinal cord. In addition, the interaction between a CPG and sensory input is important for gait generation. Attempts have been made to enhance proprioceptive sensory input to the CPG in patients whose walking was assisted on a treadmill in order to improve the effect of rehabilitation (Dietz 2002). In recent years, it has been reported that independent gait could be restored in complete paraplegia by training using a method of functional electrical stimulation that stimulates spatially selective regions of the lumbar spinal cord, performed with timing that coincided with the intended movement (Gill et al. 2018; Wagner et al. 2018).

Looking at the results of these studies, we cannot find anything that contradicts the global entrainment hypothesis that human gait is generated by the dynamic interaction between the neural system including the CPG, the body, and the environment, even 30 years after the proposal. However, there is still debate about the magnitude of relative contributions of the higher center, CPG, and sensory input when gait is generated (Minassian et al. 2017; Klarner and Zehr 2018). Gaining complete proof may be difficult unless we can directly verify the workings of all the elements of the neural and musculoskeletal system when walking in a real environment. A large-scale computer simulation incorporating details of the neuromuscular system will provide an important clue to understanding the dynamics of human locomotion in future studies (Ogihara and Yamazaki 2001; De Groote and Falisse 2021).

Another approach to elucidate the mechanisms of human walking is to scrutinize the developmental processes of walking. It is well known that neonates produce gait-like stepping movements when held upright, which may be a manifestation of locomotor CPG activity. Dominici et al. (2011) analyzed the muscle activity pattern during stepping and demonstrated that neonates showed an alternating pattern of extensors and flexors, whereas toddlers, preschoolers, and adults showed a pattern with four rhythmic components with phase differences. This supports Taga's model (1995a), which had four rhythm generator units, and phase differences were stabilized by the interaction between the spinal CPG and sensory input. In recent years, motoneuron activity in the spinal cord of neonates has been estimated using high-density EMG electrodes (Del Vecchio et al. 2020). Further development of technology for noninvasive recording of neural activity will deepen our understanding of the mechanism of gait by clarifying the details of the developmental process from the fetal period to infancy.

\section{Origin of spontaneous activity}

After conducting a series of studies on modeling human locomotion, I thought that I had done almost everything I could with the computer simulation. To study more fundamental issues, I shifted the focus of my research to the development of motion and perception in human infants. In particular, I 
pursued the establishment of noninvasive brain imaging as functional near-infrared spectroscopy (fNIRS), which can be applied to infants.

A fundamental issue that remains to be solved is the origin of rhythm in the spontaneous activity of the brain. While neurons have the capability to produce oscillatory activity, energy supply and metabolism are essential for neural activity to occur. It should be noted that rhythm generation in metabolism, such as glycolysis, has long attracted the attention of theoretical studies in terms of self-organization (Goldbeter 2002). In recent years, systems biology, with the help of computer science, has increasingly contributed to understanding the vast metabolic pathways at the system level. However, limited information is available regarding the dynamic interaction between heterogeneous systems such as the vascular, metabolic, and neural systems (Chander and Chakravarthy 2012). Our group conducted fNIRS studies of the brain in infants and showed that a relative phase between oscillatory changes in physiological parameters is a good indicator of the development of the vascular, metabolic, and neural systems (Watanabe et al. 2017). There seem to be many old and new questions about how metabolism contributes to rhythm generation in the brain.

The spatiotemporal activity of the brain dynamically changes as neural networks develop from the fetal to infancy periods. In the last 20 years, advances in noninvasive brain imaging techniques such as magnetic resonance imaging (MRI) and NIRS have revealed how neural activity emerges and how the structural and functional networks are generated in the developing brain (Vasung et al. 2019). Our group contributed to the first report of spontaneous hemodynamic changes in the newborn cortex using fNIRS (Taga et al. 2000). The spontaneous hemodynamic changes observed by fNIRS provide rich information to reveal the development of largescale cortical networks in the first 6 months of life (Homae et al. 2010). We also showed that many regions of the cortex, in relation to vision, audition, language, and learning, function by 2-3 months of age (Taga et al. 2003, 2018; Homae et al. 2006; Watanabe et al. 2008; Nakano et al. 2009). In recent years, imaging studies on preterm infants and fetuses have progressed, and the understanding of the developmental mechanisms of the brain is deepening at the macroscopic level. It should be noted that the current imaging techniques have limitations in observing spatiotemporal neural activity at the microscopic level. Thus, we need to pursue a novel imaging technique in order to understand the developmental mechanisms for information generation in the brain on multiple scales. I feel that the time is coming when it will be possible to create and simulate a large-scale model for the developing neural system with the body, as well as an environment that verifies the developmental mechanism of the emergence of behaviors.

\section{Emergence of motor behaviors}

Cybernetics has constructed a framework for understanding the neural mechanisms underlying human behavior. However, it is not yet clear how the intention of behavior and the sense of self-agency emerge. Most studies on motor control have been conducted under the condition that a goal of behavior is given. In order to overcome this difficulty, it is necessary to pursue the developmental principle that the brain actively interacts with the environment, beginning from spontaneous fetal movements.

Ultrasound measurements show that the first movement appears at approximately 7.5 weeks of gestational age (Einspieler et al. 2021). Spontaneous movement in the early fetal period is thought to be caused by spontaneous activity of the spinal cord, including the CPG. Importantly, the initial movements are spontaneous movements, not due to sensory stimuli or reflexes, and sensory inputs are formed later. Furthermore, the initial activity in the developing cortex is generated in subplate neurons, which are transient neurons that appear in the fetal period and are involved in the network formation of the cortex (Molnár et al. 2020). While it is not clear how the interaction between the cortex and subcortex, including the CPG and sensory pathways, is established in the fetus, the subplate neurons may be involved in promoting locomotor activity by the brainstem-spinal cord system and somatotopy of the cerebral cortex by sensory inputs generated by locomotor activity. More interestingly, the degeneration of the subplates coincides with the timing of when the spontaneous general movements seen in the neonatal period disappear after the first few months of life and intentional movements develop (Hadders-Algra 2018). Despite the transient properties of spontaneous general movements, we gathered evidence to show that alternation of the patterns of general movements in early infancy is related to motor and cognitive development later on (Kanemaru et al. 2013; Gima et al. 2018). Therefore, the subplates are supposed to play a key role in integrating subcortical spontaneous movements to create intentional movements that are controlled primarily by the cortex.

After birth, infants are exposed to sensory stimuli. An interesting question is whether spontaneous movements are modulated by sensory signals. Our group found that spontaneous stepping movements of young infants in the supine position were synchronized with musical rhythms (Fujii et al. 2014). This suggests that the nonlinear oscillatory activity of the spinal circuit for generating stepping movements can be entrained to the cortical rhythmic activity induced by the auditory system.

For the first few months of life, infants gradually exhibit goal-directed movements through interactions with the novel environment. A fundamental question is how the sense of agency is acquired and how the voluntary control of movement is established. To tackle this issue, our group explored 
infants' behavioral changes in a novel environment (Watanabe and Taga 2006). When an infant was brought into an environment where a mobile toy was hung and the arm was tied to a mobile, the infant increased the movements of the toy by increasing their body movements, thereby generating global entrainment between spontaneous movements and environmental changes. While younger infants moved all the limbs, older infants selectively moved the arm attached to the toy. Even if the string was cut, the infants remembered the previous action and tried to replay it. More interestingly, in 3-month-old infants, when the mobile was moved by another person, they suppressed their motor activity and concentrated on observing the mobile toy (Watanabe et al. 2011). These behavioral changes are thought to indicate the development of a sense of agency and the emergence of goal-directed movements. Most previous studies on the neural mechanisms for consciousness and intentionality were conducted in adults, and they have limited scope to solve these problems. I believe that the emergence of spontaneous activity during development is a necessary condition for consciousness and intentionality.

\section{From emergence of spontaneous activity to coexistence in a world}

Rhythm is a universal phenomenon not only in the brain, but also in life. It has been a long time since a reaction-diffusion system was proposed as a model for morphogenesis by extending the pioneering theory of pattern formation by Turing (Gierer and Meinhardt 1972). It is relatively recent that various rhythmic phenomena have been reported in gene expression during development, and gene oscillators are being given a firm role as a developmental mechanism (Morelli et al. 2012). Although the findings of animal research do not always apply to the understanding of human development, modeling research is also considered to play an important role. In conventional theoretical biology, understanding morphogenesis is a goal of research, but in the future, it will be necessary to build a model that includes functional expression in combination with pattern formation. Recently, the structural development of the human brain on a macroscopic scale has been understood in terms of growing materials and fibers (Van Essen 2020). Neural mechanisms should be pursued along with the construction of brain morphology and networks. Such an approach will open the way to understanding the design principle for biological systems in general and humans in particular. In that sense, developmental biology and neuroembryology are involved in control and information issues more than ever before.

Problems with control and information at the individual level inevitably involve interactions with other individuals and the environment. While mathematical biology has con- tributed to modeling population dynamics by simplifying the characteristics of individuals, cybernetics needs to take into account the complexity of both internal and external dynamics, thereby revealing the development of communication, language, and symbiosis on Earth. Rethinking the question posed by Taga et al. (1991), how can biological systems, including humans, generate adaptive behavior in unpredictable environments? The brain is not a pattern classification machine for information coming from the outside world, but a system that interacts with an unknown environment by spontaneously creating information and acting on the environment. Development is a phenomenon that establishes embodiment, endlessly creates internal complexity, and at the same time, enables coexistence with complex environments. Symbiosis with the gut microbiota (Gilbert et al. 2012) and possible contributions of the microbiota to the development of the human brain should be a new study field.

Biological Cybernetics is the oldest journal devoted to theories about information processing and control in living organisms. The issues to be pursued will change from time to time because of innovation with new measurement technologies and analysis methods, but the vision of system theory that Biological Cybernetics has set forth is universal. In the age of big data, it is important to unravel how biological systems, including humans, survive in an unknown environment where no previous data are available.

Acknowledgements This work was partly supported by JSPS Grant-inAid for Scientiic Research Number 16H06525, 19H01086, 19KK0247 and 20K20601.

\section{References}

Amari SI (1977) Neural theory of association and concept-formation. Biol Cybern 26(3):175-185

Asada M, Hosoda K, Kuniyoshi Y, Ishiguro H, Inui T, Yoshikawa Y, Yoshida C (2009) Cognitive developmental robotics: a survey. IEEE Trans Auton Ment Dev 1(1):12-34

Chander BS, Chakravarthy VS (2012) A computational model of neuroglio-vascular loop interactions. PLOS ONE 7(11):e48802

De Groote F, Falisse A (2021) Perspective on musculoskeletal modelling and predictive simulations of human movement to assess the neuromechanics of gait. Proc Biol Sci 288(1946):20202432

de Rugy A, Taga G, Montagne G, Buekers MJ, Laurent M (2002) Perception-action coupling model for human locomotor pointing. Biol Cybern 87(2):141-150

Del Vecchio A, Sylos-Labini F, Mondì V, Paolillo P, Ivanenko Y, Lacquaniti F, Farina D (2020) Spinal motoneurons of the human newborn are highly synchronized during leg movements. Sci Adv 6(47):eabc3916

Dietz V (2002) Proprioception and locomotor disorders. Nat Rev Neurosci 3(10):781-790

Dimitrijevic MR, Gerasimenko Y, Pinter MM (1998) Evidence for a spinal central pattern generator in humans. Ann N Y Acad Sci 860(1):360-376 
Dominici N, Ivanenko YP, Cappellini G, d'Avella A, Mondì V, Cicchese M, Lacquaniti F (2011) Locomotor primitives in newborn babies and their development. Science 334(6058):997-999

Drew T (1988) Motor cortical cell discharge during voluntary gait modification. Brain Res 457(1):181-187

Einspieler C, Prayer D, Marschik PB (2021) Fetal movements: the origin of human behaviour. Dev Med Child Neurol 63:1142

Ekeberg Ö (1993) A combined neuronal and mechanical model of fish swimming. Biol Cybern 69(5-6):363-374

Freeman WJ (1987) Simulation of chaotic EEG patterns with a dynamic model of the olfactory system. Biol Cybern 56(2-3):139-150

Fujii S, Watanabe H, Oohashi H, Hirashima M, Nozaki D, Taga G (2014) Precursors of dancing and singing to music in three- to four-months-old infants. PLOS ONE 9(5):e97680

Fukuoka Y, Kimura H, Cohen AH (2003) Adaptive dynamic walking of a quadruped robot on irregular terrain based on biological concepts. Int J Robot Res 22(3-4):187-202

Gierer A, Meinhardt H (1972) A theory of biological pattern formation. Kybernetik 12(1):30-39

Gilbert SF, Sapp J, Tauber AI (2012) A symbiotic view of life: we have never been individuals. Q Rev Biol 87(4):325-341

Gill ML, Grahn PJ, Calvert JS, Linde MB, Lavrov IA, Strommen JA, Zhao KD (2018) Neuromodulation of lumbosacral spinal networks enables independent stepping after complete paraplegia. Nat Med 24(11):1677-1682

Gima H, Kihara H, Watanabe H, Nakano H, Nakano J, Konishi Y, Taga G (2018) Early motor signs of autism spectrum disorder in spontaneous position and movement of the head. Exp Brain Res 236(4):1139-1148

Goldbeter A (2002) Computational approaches to cellular rhythms. Nature 420(6912):238-245

Gray CM, Singer W (1989) Stimulus-specific neuronal oscillations in orientation columns of cat visual cortex. Proc Natl Acad Sci USA 86(5):1698-1702

Grillner S (1985) Neurobiological bases of rhythmic motor acts in vertebrates. Science 228(4696):143-149

Hadders-Algra M (2018) Neural substrate and clinical significance of general movements: an update. Dev Med Child Neurol 60(1):39-46

Haken H, Kelso JA, Bunz H (1985) A theoretical model of phase transitions in human hand movements. Biol Cybern 51(5):347-356

Homae F, Watanabe H, Nakano T, Asakawa K, Taga G (2006) The right hemisphere of sleeping infant perceives sentential prosody. Neurosci Res 54(4):276-280

Homae F, Watanabe H, Otobe T, Nakano T, Go T, Konishi Y, Taga G (2010) Development of global cortical networks in early infancy. J Neurosci 30(14):4877-4882

Hopfield JJ, Tank DW (1985) 'Neural' computation of decisions in optimization problems. Biol Cybern 52(3):141-152

Ijspeert AJ (2014) Biorobotics: using robots to emulate and investigate agile locomotion. Science 346(6206): 196-203

Kaneko K (1990) Clustering, coding, switching, hierarchical ordering, and control in a network of chaotic elements. Phys D 41(2): $137-172$

Kanemaru N, Watanabe H, Kihara H, Nakano H, Takaya R, Nakamura T, Konishi Y (2013) Specific characteristics of spontaneous movements in preterm infants at term age are associated with developmental delays at age 3 years. Dev Med Child Neurol 55(8):713-721

Kawato M, Furukawa K, Suzuki R (1987) A hierarchical neuralnetwork model for control and learning of voluntary movement. Biol Cybern 57(3):169-185

Kelso JAS (2021) The Haken-Kelso-Bunz (HKB) model: from matter to movement to mind. Biol Cybern 115:305-322

Kimura S, Yano M, Shimizu H (1993) A self-organizing model of walking patterns of insects. Biol Cybern 69(3):183-193
Klarner T, Zehr EP (2018) Sherlock Holmes and the curious case of the human locomotor central pattern generator. J Neurophysiol 120(1):53-77

Kohonen T (1982) Analysis of a simple self-organizing process. Biol Cybern 44(2):135-140

Minassian K, Hofstoetter US, Dzeladini F, Guertin PA, Ijspeert A (2017) The human central pattern generator for locomotion: does it exist and contribute to walking? Neuroscientist 23(6):649-663

Miyakoshi S, Taga G, Kuniyoshi Y, Nagakubo A (2000) Threedimensional bipedal stepping motion using neural oscillators-towards humanoid motion in the real world. J Robot Soc Jpn 18(1):87-93

Molnár Z, Luhmann HJ, Kanold PO (2020) Transient cortical circuits match spontaneous and sensory-driven activity during development. Science. https://doi.org/10.1126/science.abb2153

Morelli LG, Uriu K, Ares S, Oates AC (2012) Computational approaches to developmental patterning. Science 336(6078):187-191

Nakano T, Watanabe H, Homae F, Taga G (2009) Prefrontal cortical involvement in young infants' analysis of novelty. Cereb Cortex 19(2):455-463

Ogihara N, Yamazaki N (2001) Generation of human bipedal locomotion by a bio-mimetic neuro-musculo-skeletal model. Biol Cybern 84(1):1-11

Patla AE, Prentice SD, Robinson C, Neufeld J (1991) Visual control of locomotion: strategies for changing direction and for going over obstacles. J Exp Psychol Hum Percept Perform 17(3):603-634

Pfeifer R, Scheier C (2001) Understanding intelligence. MIT Press

Raibert MH (1986) Legged robots that balance. MIT Press

Shimizu H, Yamaguchi Y, Tsuda I, Yano M (1985) Pattern recognition based on holonic information dynamics: towards synergetic computers. In: Springer series in synergetics. Springer, Berlin, pp 225-239

Shimizu H, Yamaguchi Y (1987) Synergetic computer and holonicsinformation dynamics of a semantic computer. Phys Scr 36(6):970-985

Taga G (1995) A model of the neuro-musculo-skeletal system for human locomotion. I. Emergence of basic gait. Biol Cybern 73(2):97-111

Taga G (1995) A model of the neuro-musculo-skeletal system for human locomotion. II. Real-time adaptability under various conditions. Biol Cybern 73(2):113-121

Taga G (1998) A model of the neuro-musculo-skeletal system for anticipatory adjustment of human locomotion during obstacle avoidance. Biol Cybern 78(1):9-17

Taga G, Yamaguchi Y, Shimizu H (1991) Self-organized control of bipedal locomotion by neural oscillators in unpredictable environment. Biol Cybern 65(3):147-159

Taga G, Konishi Y, Maki A, Tachibana T, Fujiwara M, Koizumi H (2000) Spontaneous oscillation of oxy- and deoxy-hemoglobin changes with a phase difference throughout the occipital cortex of newborn infants observed using non-invasive optical topography. Neurosci Lett 282(1-2):101-104

Taga G, Asakawa K, Maki A, Konishi Y, Koizumi H (2003) Brain imaging in awake infants by near-infrared optical topography. Proc Natl Acad Sci USA 100(19):10722-10727

Taga G, Watanabe H, Homae F (2018) Developmental changes in cortical sensory processing during wakefulness and sleep. Neuroimage 178:519-530

Tsuda I (1992) Dynamic link of memory - chaotic memory map in nonequilibrium neural networks. Neural Netw 5(2):313-326

Van Essen DC (2020) A 2020 view of tension-based cortical morphogenesis. Proc Natl Acad Sci USA 117(52):32868-32879

Vasung L, Abaci Turk EA, Ferradal SL, Sutin J, Stout JN, Ahtam B, Grant PE (2019) Exploring early human brain development with structural and physiological neuroimaging. Neuroimage 187:226-254 
Von Der Malsburg C, Schneider W (1986) A neural cocktail-party processor. Biol Cybern 54(1):29-40

Wagner FB, Mignardot JB, Le Goff-Mignardot CG, Demesmaeker R, Komi S, Capogrosso M, Courtine G (2018) Targeted neurotechnology restores walking in humans with spinal cord injury. Nature 563(7729):65-71

Watanabe H, Taga G (2006) General to specific development of movement patterns and memory for contingency between actions and events in young infants. Infant Behav Dev 29(3):402-422

Watanabe H, Homae F, Nakano T, Taga G (2008) Functional activation in diverse regions of the developing brain of human infants. Neuroimage 43(2):346-357

Watanabe H, Homae F, Taga G (2011) Developmental emergence of self-referential and inhibition mechanisms of body movements underling felicitous behaviors. J Exp Psychol Hum Percept Perform 37(4):1157-1173

Watanabe H, Shitara Y, Aoki Y, Inoue T, Tsuchida S, Takahashi N, Taga $G$ (2017) Hemoglobin phase of oxygenation and deoxygenation in early brain development measured using fNIRS. Proc Natl Acad Sci USA 114(9):E1737-E1744
Wiener N (1961) Cybernetics or control and communication in the animal and the machine, 2nd edn. MIT Press, London

Winter DA (1991) Biomechanics and motor control of human gait: normal, elderly and pathological. University of Waterloo Press, Waterloo

Yabe Y, Taga G (2008) Treadmill locomotion captures visual perception of apparent motion. Exp Brain Res 191(4):487-494

Yamaguchi Y, Kometani K, Shimizu H (1981) Self-synchronization of nonlinear oscillations in the presence of fluctuations. J Stat Phys 26(4):719-743

Publisher's Note Springer Nature remains neutral with regard to jurisdictional claims in published maps and institutional affiliations. 